\title{
Long-term follow-up of the use of maintenance antibiotic therapy for chronic antibiotic-dependent pouchitis
}

\author{
Jonathan P Segal, ${ }^{1,2}$ Stephanie X Poo, ${ }^{3}$ Simon D McLaughlin, ${ }^{4}$ \\ Omar D Faiz, ${ }^{1,2}$ Susan K Clark, ${ }^{1,2}$ Ailsa L Hart ${ }^{1,2}$
}

\author{
${ }^{1}$ St Mark's Hospital, Harrow, UK \\ ${ }^{2}$ Department of Surgery and \\ Cancer, Imperial College, London, \\ UK \\ ${ }^{3}$ Imperial Healthcare NHS Trust, \\ London, UK \\ ${ }^{4}$ Department of \\ Gastroenterology, The Royal \\ Bournemouth and Christchurch \\ Hospitals, Bournemouth, UK
}

\section{Correspondence to}

Dr Jonathan P Segal, St. Mark's Hospital, Harrow HA1 3UJ, UK; jonathansegal@doctors.org.uk

Received 6 October 2017 Revised 19 December 2017

Accepted 14 January 2018

Published Online First

31 January 2018
Check for updates

To cite: Segal JP, Poo SX, McLaughlin SD, et al. Frontline Gastroenterology 2018;9:154-158.

\section{ABSTRACT}

Objective Restorative proctolectomy is considered the procedure of choice in patients with ulcerative colitis who have failed medical therapy. Chronic pouchitis occurs in $10 \%-15 \%$ of patients, which often require long-term antibiotics to alleviate symptoms. Safety and efficacy of long-term maintenance antibiotics for chronic pouchitis has yet to be established. We aimed to assess the long-term safety and efficacy of maintenance antibiotic therapy for chronic pouchitis.

Design This was an observational study. We followed up patients who were diagnosed with chronic antibiotic-dependent pouchitis. Setting Data were collected from our single specialist pouch centre.

Patients Patients with chronic antibioticdependent pouchitis who had been maintained on antibiotics continuously for at least 1 year with a least one follow-up visit. Main outcome measure Development of pouch failure defined by the need for an ileostomy, patient-reported side effects of antibiotics and development of antibiotic resistance found on stool coliform testing.

Results Long-term use of antibiotics achieve remission in $21 \%$ of patients over a median follow-up of 102 (range 9-125). Pouch failure in association with chronic pouchitis after a median follow-up of 8.5 years occurred in $18 \%$. Side effects of long-term antibiotic use occurred in $28 \%$ of patients, with resistance to antibiotics from at least one stool sample occurring in $78 \%$ patients

Conclusions Although the use of antibiotics in chronic pouchitis may be justified, the use of long-term antibiotics must be weighed against potential complications associated with pouchitis and antibiotics.

\section{INTRODUCTION Background}

Restorative proctolectomy with ileal pouch-anal anastomosis is considered the procedure of choice in patients with ulcerative colitis who have failed medical therapy, as it avoids the need for a longterm ileostomy and restores intestinal continuity.

Complications can occur, with pouchitis being the most common long-term problem. The incidence of acute pouchitis is $20 \%$ at 1 year and up to $40 \%$ at 5 years. ${ }^{1}$ Pouchitis is clinically characterised by symptoms that include increased stool frequency, haematochezia, abdominal cramping, urgency, tenesmus, incontinence, fever and extraintestinal manifestations. ${ }^{2}$

The diagnosis of pouchitis has no standard definition. The most commonly used criterion is the pouch disease activity index (PDAI). The PDAI is a composite score including clinical symptoms, endoscopic findings and histological findings. A score greater than 7 is required for a diagnosis of pouchitis (see online supplementary material). ${ }^{3}$

The first-line treatment of acute pouchitis is empirical with antibiotics. Ciprofloxacin and metronidazole are the most commonly used, often generating a rapid dramatic response. ${ }^{4-7}$ Chronic pouchitis has no standard definition, but is often defined as the need for antibiotics for greater than 4 weeks with evidence of pouchitis. ${ }^{8}$ Chronic pouchitis develops in approximately $10 \%-15 \%$ of patients with acute pouchitis and can be 'treatment responsive' or 'treatment refractory' to antibiotic therapy. ${ }^{9} 10$ 
In our practice, antibiotics are often needed to maintain symptomatic response and symptoms return on withdrawal. This often results in patients being maintained on antibiotics long-term. While antibiotics are the mainstay of treatment, there are currently no data on the efficacy and long-term safety implications of using long-term antibiotics in chronic pouchitis. In particular, the use of ciprofloxacin has been associated with tendon rupture ${ }^{11}$ metronidazole with peripheral neuropathy and various antibiotic combinations have been implicated in Clostridium difficile diarrhoea. To the best of our knowledge, this is the first long-term study to explore these complications and effectiveness of long-term antibiotics for the treatment of chronic pouchitis.

\section{Objectives}

- We assessed the time from diagnosis of chronic pouchitis to pouch failure and in addition time to development of antibiotic resistance.

- We also assessed adverse effects of antibiotics that occurred as well as the development of resistant organisms.

\section{METHODS}

\section{Study design}

This was an observational study. We followed up patients who were previously diagnosed with chronic antibiotic-dependent pouchitis and whose outcomes were reported in our publication in 2009. ${ }^{12}$ Data were collected from our single centre, which is a specialist centre for both pouch surgery and a tertiary centre for pouch referrals.

\section{Participants}

Patients were included if they met all of the following inclusion criteria:

- Aged $\geq 18$ years.

- Chronic antibiotic-dependent pouchitis that had been maintained on antibiotics continuously for at least 1 year.

- PDAI score $\geq 7$ at diagnosis.

- Patients who had at least one clinic follow-up.

Patients were excluded if they met the following criteria:

> Patients with no follow-up data.

- Patients with established Crohn's disease at start of antibiotic treatment.

A further 14 patients were identified during this study period and were also included.

\section{Variables}

We followed these patients up until last clinic appointment or until pouch failure defined by the need for an ileostomy or pouch excision. Data collected included pouch failure, pouch-related complications, development of antibiotic resistance, development of Clostridium. difficile, tendon rupture, endoscopic and histological findings, and need for escalation.

\section{Measurement of variables}

We defined chronic antibiotic-dependent pouchitis as the need for antibiotics for at least 1 month to control pouchitis symptoms (defined as a PDAI greater than 7) or the use of antibiotics on three separate occasions to control pouchitis symptoms within a 1-year period. Remission was defined as a PDAI of less than 7 on follow-up. Endoscopic scores were standardised using the PDAI. Stool coliform testing to identify antibiotic sensitivities and resistance was performed as described previously. ${ }^{13}$ We assessed the time from diagnosis of chronic pouchitis to pouch failure and in addition time to development of antibiotic resistance. We also assessed adverse effects of antibiotics that occurred as well as the development of resistant organisms.

Medical records, endoscopic and histopathological reports were independently reviewed by two authors (JPS and SXP). Baseline parameters collected included patient demographics, risk factors for pouchitis (a history of smoking, extraintestinal manifestations, primary sclerosing cholangitis), symptoms (as reported by the patient) and mean stool frequency at baseline and at last follow-up.

Follow-up data recorded were mean stool frequency at last follow-up, pouch failure as defined by need for an ileostomy, presence or absence of pouchitis, side effects of antibiotics, development of resistance to antibiotics and the development of Clostridium. difficile.

\section{Study size}

We retrospectively analysed 39 patients with chronic antibiotic-dependent pouchitis at a tertiary referral centre between January 2005 and April 2017. A further 14 patients were identified during this study period and were also included. Additional patients were identified through electronic interrogation of our endoscopy database and patient notes using keywords to include 'pouchitis', 'inflammation' and 'chronic pouchitis'. Manual searches were also performed on surgical logbooks and nurse led pouch clinic letters to highlight any additional patients.

\section{Statistical methods}

Continuous variables were compared using Student's t-test with categorical variables being compared using the $\chi^{2}$ test. A binomial probability test was used to evaluate differences between our data and the reported incidence in the literature. Statistical significance was defined as $\mathrm{P}$ value $<0.05$. Statistical tests were performed using the STATA (StataCorp, College Station, Texas, USA).

\section{RESULTS}

\section{Participants and descriptive data}

Patients' characteristics, presenting symptoms and associated risk factors are outlined in table 1. The median follow-up was 102 months (range 9-125). 
Table 1 Patient characteristics, presenting symptoms and associated risk factors

\begin{tabular}{lc}
\hline Variable & Total (\%) \\
\hline Total & $39(100)$ \\
\hline Men & $30(77)$ \\
\hline Women & $9(23)$ \\
\hline Median age at presentation (years) & $42(\mathrm{IQR})(33-54)$ \\
\hline Diagnoses & \\
\hline Ulcerative colitis & $37(95)$ \\
\hline Familial adenomatous polyposis & $2(5)$ \\
\hline Presenting symptoms & \\
\hline Frequency & $26(70)$ \\
\hline Abdominal pain & $27(73)$ \\
\hline Perianal bleeding & $6(16)$ \\
\hline Urgency & $10(27)$ \\
\hline Bloating & $6(16)$ \\
\hline Incontinence & $9(24)$ \\
\hline Perianal discomfort & $7(19)$ \\
\hline Median 24-hour stool frequency (range) & $10(4-25)$ \\
\hline Risk factors & \\
\hline Smoking & 0 \\
\hline Primary sclerosing cholangitis & $1(3)$ \\
\hline Extraintestinal manifestations & $1(3)$ \\
\hline
\end{tabular}

\section{Remission versus failure rate}

Of 39 patients, 8 (21\%) achieved symptomatic remission as defined by no longer requiring antibiotics while $7(18 \%)$ developed pouch failure, over a median duration of 6 years (range 3-9).

\section{Antibiotics}

Twenty-seven (69\%) patients remained on antibiotics at last follow-up; when excluding those who had pouch failure, $27 / 32$ patients (84\%) who still had their pouch in continuity remained on antibiotics. The most common antibiotics used were ciprofloxacin (30\%), metronidazole (19\%) or a combination of both (11\%). The other antibiotics included colistin, rifaximin and cefalexin. .

A proportion of patients had tried other therapies to achieve clinical remission: biologics $(\mathrm{n}=3,8 \%)$, steroids $(n=10,26 \%)$, probiotics (VSL\#3) $(n=3,8 \%)$, elemental diet $(\mathrm{n}=13,33 \%)$ and faecal transplantation $(n=3,8 \%)$.

\section{OUTCOMES}

Median stool frequency was unchanged from baseline at 10 per 24 hours.

\section{Complications}

Complications developed in a proportion of patients: fistula $(n=7,18 \%)$, perianal sepsis $(n=6,15 \%)$ and incontinence $(n=5,16 \%)$.

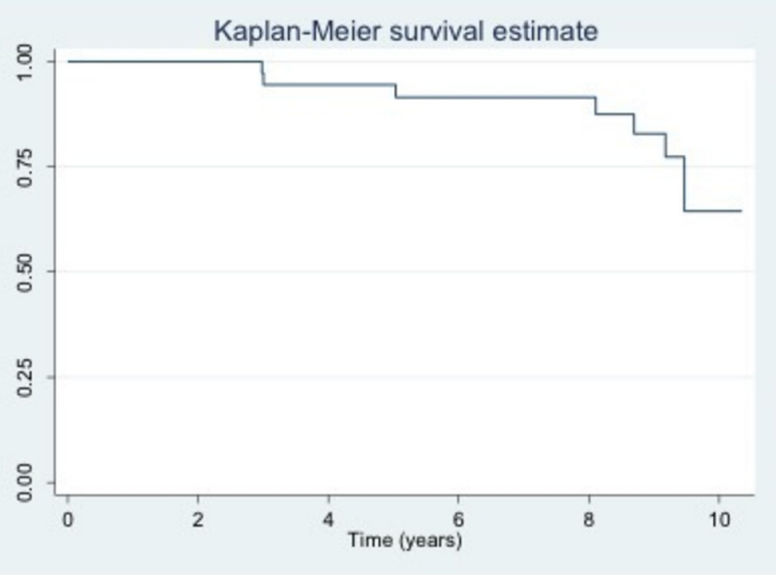

Figure 1 Kaplan-Meier survival estimate-pouch survival on long term-antibiotics.

\section{Hospital admissions}

Thirty-six pouch-related hospital admissions occurred in 11/39 (28\%) patients. Reasons for admission included 23 admissions for obstructive symptoms in five patients, 11 elective examinations under anaesthetic in three patients, one developed incontinence and one developed an episode of bleeding from pouch.

\section{Remission versus failure rate}

Of 39 patients, 8 (21\%) achieved symptomatic remission as defined by no longer requiring antibiotics while 7 (18\%) developed pouch failure, over a median duration of 6 years (range 3-9) (figure 1).

\section{Endoscopic examination}

Mean number of pouchoscopies performed was seven (range 0-19). Of all findings, the only significant change from initial to final pouchoscopy was the loss of vascular pattern $\left(\chi^{2}=7.5165, \mathrm{P}=0.006\right)$.

\section{Initial endoscopic findings}

At initial endoscopy where antibiotics were initiated, $15(39 \%)$ of patients had ulcers, $8(21 \%)$ of patients had a loss of vascular pattern, $5(14 \%)$ of patients had pre-pouch ileitis, 5 (14\%) had mucus exudate/oedema, $2(4 \%)$ had erythema, $2(4 \%)$ had friability and $2(4 \%)$ had inflammation around the cuff.

\section{Histological assessment}

Twenty-eight (74\%) had pouchitis identified from biopsy specimens taken at index pouchoscopy. Change in histological scores is tabulated in table 2.

The change in scores was based on the Moskowitz scoring system. ${ }^{14}$ This is based on an acute score, which encompasses polymorph infiltration and presence of ulceration, and a chronic score, which includes the presence of chronic cell infiltrates and degree of villous atrophy. Both the acute and chronic scores are graded as $0-6$. 
Table 2 Histological scores

\begin{tabular}{llll}
\hline & First scope & Last scope & P value \\
\hline $\begin{array}{l}\text { Pathologist-labelled } \\
\text { pouchitis (\%) }\end{array}$ & $28 / 38(74)$ & $13 / 38(34)$ & $<0.006$ \\
$\begin{array}{l}\text { Mean total pouchitis score } \\
\text { (range) }\end{array}$ & $5.8(1-10)$ & $4.2(1-10)$ & $<0.009$ \\
$\begin{array}{l}\text { Mean total acute scores } \\
\text { (range) }\end{array}$ & $2.3(0-6)$ & $1.5(0-5)$ & $<0.008$ \\
$\begin{array}{l}\text { Mean total chronic scores } \\
\text { (range) }\end{array}$ & $3.4(0-5)$ & $2.6(0-5)$ & $<0.04$ \\
\hline
\end{tabular}

\section{Antibiotic-related complications}

Antibiotic-associated side effects occurred in 11 patients $(28 \%)$ : the majority developed intolerance or had worsening flares on commencement of antibiotic, one developed dysgeusia and one developed peripheral neuropathy that resolved on withdrawal of metronidazole. No patient developed tendon rupture.

\section{Antibiotic resistance patterns}

Common organisms isolated from stool cultures included coliforms $(\mathrm{n}=24,65 \%)$, Escherichia coli $(\mathrm{n}=18,49 \%)$, extended spectrum beta-lactamase producing organisms $(\mathrm{n}=12,32 \%)$ and Klebsiella spp. $(\mathrm{n}=9,24 \%)$.

Antibiotic resistance was identified from at least one stool sample in 28/36 (78\%) patients. Common resistance patterns and the rate of desensitisation from available stool samples are detailed in table 3.

Clostridium difficile toxin was not detected in any stool samples.

\section{DISCUSSION}

Our data show that the long-term use of antibiotics achieve remission in only $21 \%$ of patients over a median follow-up of 102 (range 9-125). Pouch failure in association with chronic pouchitis after a median follow-up of 8.5 years occurred in $18 \%$. Side effects of long-term antibiotic use occurred in $28 \%$ of patients, with resistance to antibiotics from at least one stool sample occurring in $78 \%$ of patients.

Remission rates in chronic pouchitis are variable with a meta-analysis highlighting that an overall remission rate following antibiotic use for chronic pouchitis was $70 \% .{ }^{15}$ When using a binomial probability test, we found that significantly less of our patients achieved remission $(\mathrm{P}<0.001)$. This suggests that the long-term use of antibiotics is poorly

\begin{tabular}{|c|c|c|}
\hline Antibiotic & Resistance rate $\%$ & Re-sensitisation rate $\%$ \\
\hline Ciprofloxacin & 74 & 32 \\
\hline Co-amoxiclav & 47 & 25 \\
\hline Trimethoprim & 74 & 12 \\
\hline Nitrofurantoin & 15 & 60 \\
\hline Cefalexin & 38 & 23 \\
\hline
\end{tabular}

effective in achieving clinical remission for those patients with chronic pouchitis, but may be useful at keeping symptoms under control. It may also suggest that chronic pouchitis is a separate entity and pathogenesis from acute pouchitis where treatment success with antibiotics is as high as $90 \% .^{15}$

In our cohort, the only significant endoscopic change from initial pouchoscopy to last follow-up was in loss of vascular pattern, which significantly improved when using antibiotics $(\mathrm{P}<0.006)$. This may represent the challenges in recording endoscopic findings while also suggesting that antibiotics may not improve endoscopic findings in chronic antibiotic-dependent patients. Indeed, previous data from our unit suggest that only $50 \%$ of patients achieve mucosal healing. ${ }^{12}$

Our study highlights that antibiotics significantly improved acute and chronic as well as total histological scores from first biopsies to most recent follow-up biopsies $(\mathrm{P}<0.05)$. This suggests that antibiotics may have a positive impact at the microscopic level in patients with chronic antibiotic-dependent pouchitis. The impact of histological mucosal healing on pouch function long-term is unknown, but our data suggest that this is a poor surrogate marker as a measure of symptom-free remission, as despite histological healing only $16 \%$ of our patients achieved clinical remission in terms of avoidance of antibiotics.

The most common adverse effects associated with ciprofloxacin have been reported as gastrointestinal and neurological. ${ }^{16}$ Ciprofloxacin has been reported as being associated with achilles tendonitis, tendon rupture and peripheral neuropathies. ${ }^{11} 17$ In our cohort, no patient developed Achilles tendonitis. One patient developed peripheral neuropathy secondary to metronidazole use. Long-term antibiotic use is associated with Clostridium difficile. ${ }^{18}$ In our cohort, despite long-term antibiotics, there were no episodes of Clostridium difficile. Despite Clostridium difficile being more widely recognised in the pouch, ${ }^{19-21}$ published data suggest that it is still a rare occurrence with one study reporting prior antibiotic use was not a strong predictor of developing Clostridium difficile. ${ }^{19}$ Despite the lack of side effects reported in our data, it is likely that with larger numbers of patients treated, many of these side effects will be found.

In terms of patterns of antibiotic resistance, ciprofloxacin and trimethoprim had high rates of resistance with 25/34 (74\%) patients developing resistance. Interestingly, resistance to antibiotics did not appear to be persistent with 8/25 (32\%) patients in the ciprofloxacin group becoming re-sensitised to ciprofloxacin on subsequent stool samples without intervention.

\section{Limitations}

This study has a number of limitations. It is a retrospective analysis from a single institution with variability in endoscopic and histopathological reporting. Our study is further limited by small patient numbers, which may account for type 2 errors, as well as limited accounts of 
potential risk factors, symptomatology and change in antibiotics for each patient. Furthermore, patients developed other problems during the follow-up period, which could account for some of the symptomatology.

\section{CONCLUSION}

The long-term use of antibiotics is poorly effective in achieving clinical remission where treatment is no longer needed to control symptoms. Long-term use of antibiotics for chronic pouchitis is associated with development of antibiotic-related side effects and development of antibiotic resistance.

Although the use of antibiotics in chronic pouchitis may be justified, the utility of long-term antibiotics must be weighed against potential complications associated with pouchitis and antibiotics. Future work will benefit from prospective multi-institutional data collection, and the development of risk stratification tools for pouch survival in selected groups of patients.

\section{Significance of this study}

\section{What is already known on this topic}

Chronic pouchitis occurs in $10 \%-15 \%$ of patients, which often require long-term antibiotics to alleviate symptoms. Safety and efficacy of long-term maintenance antibiotics for chronic pouchitis has yet to be established in these patients.

\section{What this study adds}

The long-term use of antibiotics are poorly effective in achieving remission. Long-term use of antibiotics for chronic pouchitis is associated with development of antibioticrelated side effects in a third of patients and development of antibiotic resistance in three quarters of patients.

\section{How might it impact on clinical practice in the foreseeable future}

Although the use of antibiotics in chronic pouchitis may be justified, the use of long-term antibiotics must be weighed against potential complications associated with pouchitis and antibiotics.

Contributors Guarantor of the article: ALH. JPS and SXP collected, managed and analysed the data. JPS, SXP and SDM reviewed the literature and prepared the manuscript. JPS, SXP, SDM, ALH, SKC and ODF have revised the manuscript critically and prepared the final version of the manuscript. All authors approved the final draft prior to submission.

Funding This research received no specific grant from any funding agency in the public, commercial or not-for-profit sectors.

Competing interests None declared.

Provenance and peer review Not commissioned; externally peer reviewed.

(C) Article author(s) (or their employer(s) unless otherwise stated in the text of the article) 2018. All rights reserved. No commercial use is permitted unless otherwise expressly granted.

\section{REFERENCES}

1 Lepistö A, Luukkonen P, Järvinen HJ. Cumulative failure rate of ileal pouch-anal anastomosis and quality of life after failure. Dis Colon Rectum 2002;45:1289-94.
2 Sandborn WJ. Pouchitis: definition, risk factors, frequency, natural, history, classification, and public health perspective. In: McLeod RS, Martin F, Sutherland LR, Wallace JL, Williams CN. eds. Trends in inflammatory bowel disease. Lancaster, England: Kluwer, 1997:51-63.

3 Sandborn WJ, Tremaine WJ, Batts KP, et al. Pouchitis after ileal pouch-anal anastomosis: a Pouchitis Disease Activity Index. Mayo Clin Proc 1994;69:409-15.

4 Madden MV, McIntyre AS, Nicholls RJ. Double-blind crossover trial of metronidazole versus placebo in chronic unremitting pouchitis. Dig Dis Sci 1994;39:1193-6.

5 Gionchetti P, Rizzello F, Venturi A, et al. Antibiotic combination therapy in patients with chronic, treatmentresistant pouchitis. Aliment Pharmacol Ther 1999;13:713-8.

6 Shen B, Achkar JP, Lashner BA, et al. A randomized clinical trial of ciprofloxacin and metronidazole to treat acute pouchitis. Inflamm Bowel Dis 2001;7:301-5.

7 Mimura T, Rizzello F, Helwig U, et al. Four-week open-label trial of metronidazole and ciprofloxacin for the treatment of recurrent or refractory pouchitis. Aliment Pharmacol Ther 2002;16:909-17.

8 Gionchetti P, Rizzello F, Poggioli G, et al. Oral budesonide in the treatment of chronic refractory pouchitis. Aliment Pharmacol Ther 2007;25:1231-6.

9 Pardi DS, Shen B. Endoscopy in the management of patients after ileal pouch surgery for ulcerative colitis. Endoscopy 2008;40:529-33.

10 Shen B. Pouchitis: what every gastroenterologist needs to know. Clin Gastroenterol Hepatol 2013;11:1538-49.

11 Wise BL, Peloquin C, Choi H, et al. Impact of age, sex, obesity, and steroid use on quinolone-associated tendon disorders. Am J Med2012;125:1228.e23-1228.e28.

12 McLaughlin SD, Clark SK, Tekkis PP, et al. An open study of maintenance antibiotic therapy for chronic antibioticdependent pouchitis: efficacy, complications and outcome. Colorectal Dis 2011;13:438-44.

13 McLaughlin SD, Clark SK, Shafi S, et al. Fecal coliform testing to identify effective antibiotic therapies for patients with antibiotic-resistant pouchitis. Clin Gastroenterol Hepatol 2009; 7:545-8.

14 Shepherd NA, Jass JR, Duval I, et al. Restorative proctocolectomy with ileal reservoir: pathological and histochemical study of mucosal biopsy specimens. J Clin Pathol 1987;40:601-7.

15 Segal JP, Ding NS, Worley G, et al. Systematic review with meta-analysis: the management of chronic refractory pouchitis with an evidence-based treatment algorithm. Aliment Pharmacol Ther 2017;45:581-92.

16 Zabraniecki L, Negrier I, Vergne P, et al. Fluoroquinolone induced tendinopathy: report of 6 cases. J Rheumatol 1996;23:516-20.

17 Carroll MW, Jeon D, Mountz JM, et al. Efficacy and safety of metronidazole for pulmonary multidrug-resistant tuberculosis. Antimicrob Agents Chemother 2013;57:3903-9.

18 Brown KA, Khanafer N, Daneman N, et al. Meta-analysis of antibiotics and the risk of community-associated Clostridium difficile infection. Antimicrob Agents Chemother 2013;57:2326-32.

19 Shen BO, Jiang ZD, Fazio VW, et al. Clostridium difficile infection in patients with ileal pouch-anal anastomosis. Clin Gastroenterol Hepatol 2008;6:782-8.

20 Boland E, Thompson JS. Fulminant clostridium difficile enteritis after proctocolectomy and ileal pouch-anal anastamosis. Gastroenterol Res Pract 2008;2008:1-5.

21 Martinez Ugarte ML, Lightner AL, Colibaseanu D, et al. Clostridium difficile infection after restorative proctocolectomy and ileal pouch anal anastomosis for ulcerative colitis. Colorectal Disease 2016;18:0154-O157. 